\title{
Budesonid-Formoterol als Bedarfsmedikation reduziert Kortikoid-Dosis bei Asthma
}

O’Byrne PM et al. Inhaled Combined Budesonide-Formoterol as Needed in Mild Asthma. N Engl J Med 2018; doi:10.1056/NEJMoal1715274

Bateman ED et al. As-Needed BudesonidFormoterol versus Maintenance Budesonide in Mild Asthma. N Engl ] Med 2018; doi:10.1056/ NEJMoal1715275

Patienten mit leichtem Asthma profitieren von einer langfristigen Steroidgabe, die Compliance lässt allerdings aufgrund befürchteter Nebenwirkungen häufig zu wünschen übrig. Da gleichzeitig schnell wirksame $\beta 2-\mathrm{Mi}$ metika als sehr wirksam erlebt werden, haben nun 2 Forscherteams die Kombination aus Budesonid und Formoterol als Bedarfsmedikation mit Terbutalin und einer Budesonid-Dauertherapie verglichen.

O’Byrne et al. führten eine doppelblinde, kontrollierte und randomisierte Phase-IIStudie durch, für die sie zunächst 3849 Patienten rekrutierten, die mindestens 12 Jahre alt waren und an einer milden Form von Asthma bronchiale litten. Für eine Interventionsdauer von 52 Wochen wurden sie 1 von 3 Studiengruppen zugeordnet:

- Gruppe 1: 2-mal tgl. Dauertherapie mit Placebo und Budesonid-Formoterol $(200+6 \mu g)$ als Kombinationspräparat bei Bedarf

- Gruppe 2: 2-mal tgl. Dauertherapie mit Placebo und Terbutalin $(0,5 \mathrm{mg})$ bei Bedarf

- Gruppe 3: 2-mal tgl. Dauertherapie mit Budesonid $(200 \mu \mathrm{g})$ und Terbutalin bei Bedarf

Die Autoren wollten vor allem die Sicherheit und Wirksamkeit von BudesonidFormoterol bei Bedarf untersuchen und stellten ferner die Hypothese auf, dass diese Kombination einer Bedarfsmedikation mit Terbutalin in Hinblick auf die Symptomkontrolle überlegen sei. Um die Symptomkontrolle der Asthmapatienten objektivieren zu können, griffen die Forscher auf ein elektronisches Symptomtagebuch zurück, das die Patienten während der Studienphase regelmäßig führten. Weiterhin registrierten sie Schwere und Häufigkeit akuter Exazerbationen, zusätzlichen Bedarf an Kortikoiden sowie unerwünschte Arzneimittelwirkungen in allen Studiengruppen. In der zweiten klinischen, randomisierten und kontrollierten Studie von Bateman et al. wollten die Forscher die Nichtunterlegenheit von Budesonid-Formoterol bei Bedarf gegenüber einer Erhaltungstherapie mit Budesonid nachweisen. Sie bildeten daher 2 Studiengruppen:

- Kombinationsgruppe: 2-mal tgl. Dauertherapie mit Placebo und Budesonid-Formoterol $(200+6 \mu \mathrm{g})$ als Kombinationspräparat bei Bedarf

- Kortikoid-Gruppe: 2-mal tgl. Dauertherapie mit Budesonid $(200 \mu \mathrm{g})$ und Terbutalin bei Bedarf

Diese Gruppen entsprechen dabei Gruppe 1 und Gruppe 3 aus der Studie von O’Byrne et al., Dauer und Einschlusskriterien waren vergleichbar. Als wesentliche klinische Endpunkte definierten die Autoren ebenfalls die Symptomkontrolle, den zusätzlichen Kortikoid-Bedarf sowie Anzahl und Schwere akuter Exazerbationen.

\section{Ergebnisse}

Studie von O’Byrne et al.: 3849 Datensätze gingen in die finale Analyse mit ein, davon 1277 in Gruppe 1, 1277 in Gruppe 2 und 1282 in Gruppe 3. Patienten der Gruppe 1 zeigten eine deutlich bessere Symptomkontrolle als ihre Mitpatienten der Gruppe 2. Im Vergleich zur Gruppe 3 mit Budesonid-Dauertherapie waren sie allerdings hinsichtlich der Anzahl von symptomarmen Wochen im elektronischen Tagebuch deutlich unterlegen. Patienten unter der Kombinationstherapie als Bedarfsmedikation benötigten allerdings geringe Dosierungen an zusätzlichen Kortikoiden und meldeten auch seltener akute Exazerbationen als Teilnehmer der anderen beiden Studiengruppen.
Studie von Bateman et al.: 4176 Patienten nahmen an der Studie teil, 2089 von ihnen wurden der Kombinationsgruppe zugeordnet. In Bezug auf die Symptomkontrolle war die Bedarfsmedikation mit Budesonid-Formoterol einer Erhaltungstherapie mit Budesonid wider Erwarten der Autoren unterlegen. Die zusätzliche tägliche Dosis an Kortikoiden lag bei ihnen allerding mit $66 \mu \mathrm{g}$ deutlich niedriger als in der Kortikoid-Gruppe $(267 \mu \mathrm{g})$. In Hinblick auf die Anzahl schwerer akuter Exazerbationen konnten die Forscher anhand der erhobenen Daten die Nichtunterlegenheit der Kombinationstherapie bei Bedarf statistisch signifikant belegen.

\section{FAZIT}

Budesonid-Formoterol bei Bedarf war bei Asthmapatienten der symptomorientierten Einnahme von Terbutalin hinsichtlich der Symptomkontrolle überlegen. Obwohl es zu weniger Exazerbationen kam als unter Dauermedikation mit Budesonid mussten die Autorinnen/Autoren beider Studien in puncto Symptomkontrolle jedoch eine Unterlegenheit feststellen. Da Patienten unter der Kombination allerdings weniger Kortikoide benötigten, halten die Forscher Budesonid-Formoterol für eine sinnvolle Therapieoption.

Dipl.-Psych. Annika Simon, Hannover

\section{Kommentar}

Die Asthma-Behandlung basiert auf antientzündlichen Therapien; die wirksamste Substanzgruppe ist das inhalative Steroid (ICS). Die zentralen Therapieziele sind Symptomkontrolle, Vermeidung von Exazerbationen und Erhaltung der Lungenfunktion.

Die aktuellen Asthma-Leitlinien empfehlen in Stufe 2 den kontinuierlichen Einsatz von ICS ggf. in Kombination mit einem kurzwirksamen $\beta$-2-Sympathomi- 
metikum (SABA) [1]. Reicht dies nicht aus, wird in Stufe 3 die Dauertherapie mit einem ICS in Kombination mit einem langwirksamen $\quad \beta$-2-Sympathomimetikum (LABA) empfohlen, welche als fixe Kombination in einem Inhalations-Device verabreicht werden kann. Zusätzlich ist hier zur Erhaltungstherapie ein bedarfsorientierter Einsatz einer Formoterol-haltigen ICS/LABA-Kombination als Therapieoption möglich. Es ist bekannt, dass die Therapieadhärenz für den kontinuierlichen Einsatz von ICS gering ist, da Patienten keinen sofortigen Effekt der Inhalation spüren. Die Einnahme wird daher von stabilen Patienten häufig „vergessen“, weswegen Möglichkeiten einer flexiblen Behandlung in dieser Stufe diskutiert werden. Ältere Studien evaluieren bereits den bedarfsorientierten Einsatz von ICS oder fixen Kombinationen von ICS und SABA [2,3]. Bei bedarfsorientierter Therapie ist die Anzahl akuter Verschlechterungen (Exazerbationen) vergleichbar zur Dauertherapie mit ICS. Dies konnte mit einer deutlich reduzierten Menge an inhaliertem Steroid erreicht werden, was bedeutet, dass eine Asthmakontrolle mit einer nicht kontinuierlichen, antientzündlichen Behandlung erreicht werden kann. Im Gegensatz zum ICS spürt der Patient bei einem rasch wirksamen Bronchodilatator nach der Inhalation eine schnelle Bronchodilatation. Für eine bedarfsgesteuerte Therapie ist daher die Kombination eines ICS mit einem rasch wirksamen Bronchodilatator sinnvoll. Diese sollte in einem Device erfolgen, um eine Bronchodilatator-Monotherapie zu vermeiden.

Die vorliegenden Studien untersuchen eine Kombination von ICS und LABA als bedarfsorientierte Therapie im Vergleich zur ICS-Dauertherapie. Das hier verwendete LABA Formoterol hat einen raschen Wirkungseintritt und ist daher zur akuten Behandlung einsetzbar. Der Einsatz der flexiblen Kombination unterschied sich nicht zur Anzahl von Exazerbationen und Lungenfunktion im Vergleich zur ICS-Dauertherapie. Die Daten von O’Byrne et al. untermauern die Empfehlung, dass eine alleinige Behandlung mit Bronchodilatatoren bei Asthma kontraindiziert ist. Im Gegensatz dazu ist eine fixe Kombination von ICS mit einem
LABA sicher. Aktuelle Analysen großer Sicherheitsstudien zeigen, dass die fixe Kombination von LABA und ICS keine erhöhte Wahrscheinlichkeit für schwere Nebenwirkungen hat [4]. Die Food-andDrug-Administration (FDA) hat bisher bestehende Warnhinweise aus der Fachinformation genommen [5]. Die Kombinationen können ohne Bedenken auch bei leichtgradig erkrankten Patienten eingesetzt werden. Zusätzlich führt die bedarfsorientierte Behandlung zu einer deutlichen Reduktion der benötigten ICS-Dosis. Die vergleichbare Wirksamkeit der bedarfsorientierten Therapie zur Dauerbehandlung bei den Endpunkten Exazerbation und Lungenfunktion ist bemerkenswert. Die ICS-Dosis der flexiblen Behandlungsgruppe lag bei beiden Studien $75 \%$ oder 83 \% unterhalb der Dauerbehandlungsgruppe. Zwar sind mögliche Nebenwirkungen des ICS gering, diese Reduktion kann bei „Kortisonkritischen“ Patienten aber zur Steigerung der Therapieakzeptanz führen. Ein weiterer interessanter Aspekt ist die Kosten-Reduktion. Fixe Kombinationen sind deutlich teurer als ICS-Monopräparate; durch die deutliche Reduktion der Einnahmehäufigkeit ist aber eine Reduktion der Gesamtkosten möglich.

Beide Studien zeigen, dass die fixe Kombination aus ICS/LABA eine sichere Alternative zur herkömmlichen ICS-Dauertherapie sein kann. Gerade bei Patienten, die gerne einen größeren Anteil an der Gestaltung der Behandlung haben, ist der flexiblere Einsatz der Therapie eine gute Möglichkeit. Bisher ist der Ansatz in den Leitlinien nicht implementiert. Es ist aber zu erwarten, dass sowohl internationale als auch nationale Leitlinien diese Therapieoption in ihre Empfehlungen aufnehmen werden.

\section{Autorinnen/Autoren}

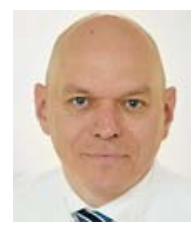

Prof. Dr. med. Christian Taube, Direktor der Klinik für Pneumologie, Universitätsmedizin Essen Ruhrlandklinik

\section{Literatur}

[1] Buhl R, Bals R, Baur X et al. S2k-Leitlinie zur Diagnostik und Therapie von Patienten mit Asthma. Pneumologie 2017; 71: e2

[2] Papi A, Canonica GW, Maestrelli P et al. Rescue use of beclomethasone and albuterol in a single inhaler for mild asthma. N Engl J Med 2007; 356: 2040-2052

[3] Boushey HA, Sorkness CA, King TS et al. Daily versus as-needed corticosteroids for mild persistent asthma. N Engl J Med 2005; 352: 1519-1528

[4] Busse WW, Bateman ED, Caplan AL et al. Combined Analysis of Asthma Safety Trials of Long-Acting beta2-Agonists. N Engl J Med 2018; 378: 2497-2505

[5] Seymour SM, Lim R, Xia C et al. Inhaled Corticosteroids and LABAs - Removal of the FDA's Boxed Warning. N Engl J Med 2018; 378 : 2461-2463 